\title{
Return to Play Following Nonoperative Treatment of Partial UInar Collateral Ligament Injuries in Professional Baseball Players: A Critically Appraised Topic
}

\author{
Nicole Cascia, Tim L. Uhl, and Carolyn M. Hettrich
}

\begin{abstract}
Clinical Scenario: Ulnar collateral ligament (UCL) injuries are highly prevalent in professional baseball players with the success of operative management being well known in the literature. Return to play (RTP) rates following nonoperative management of partial UCL injuries in professional baseball players are not well established in the literature. With a UCL tear being a potential career-ending injury, it is imperative that the best treatment option is provided to these throwing athletes. There is an increase in the incidence of UCL surgical rates and a lack of general agreement on nonoperative treatment of partial UCL injuries as reported by the American Shoulder and Elbow Surgeons in 2017. There is also a lack of clarity on when to initiate rehabilitation, which may be due to the limited amount of studies reporting success of RTP rates and time to RTP following conservative interventions of partial UCL injuries. Evidence on the RTP rates seen following conservative management of partial UCL tears injuries can help guide health care providers in deciding on the best treatment option for professional baseball athletes who desire to return to their athletic careers. These rates of RTP will add valuable objective input when determining if conservative management is the best choice. To determine the current evidence, inclusion criteria for the literature search consisted of RTP rates following conservative treatment in professional baseball players between inception and 2018. Clinical Question: Is there evidence for successful RTP rates in professional baseball players following conservative treatment of a UCL injury? Summary of Key Findings: Three retrospective studies met the inclusion criteria and were included. Of those, 2 reported RTP rates following a nonoperative rehabilitation program of a UCL injury, whereas 1 reported RTP rates after injection therapy in subjects who attempted a trial of conservative treatment. All 3 studies considered location and grade of UCL tear. Successful RTP rates (66\%$100 \%$ ) were reported in professional baseball players following nonoperative treatment of partial UCL injuries. Clinical Bottom Line: Current evidence supports high success with RTP rates up to $100 \%$ after nonoperative treatment of grade 1 UCL injuries in professional baseball players and between $66 \%$ and $94 \%$ for a grade 2 and above. Strength of Recommendation: There is level C evidence for high RTP rates following nonoperative treatment of partial UCL injuries in professional baseball players.
\end{abstract}

Keywords: rehabilitation, elbow, overhead athlete

\section{Clinical Scenario}

Numerous studies have reported on postoperative return to play (RTP) rates, between $66 \%$ and $98 \%$, in professional baseball players after ulnar collateral ligament (UCL) reconstruction. ${ }^{1-5}$ Currently, there is limited evidence following nonoperative management. There has been an increase in the incidence of UCL reconstructions performed annually, which is projected through $2025 .{ }^{6}$ A recent survey distributed to 159 active members of the American Shoulder and Elbow Surgeons assessed current trends related to the treatment of UCL injuries in athletes and found divided opinions among surgeons on how to treat partial tears. ${ }^{7}$ The decision to treat these partial tears with surgery may be due to published studies that have reported improved outcomes and RTP rates. ${ }^{5,8}$ Nonoperative treatment of UCL injuries has progressed with both preventive and rehabilitative strategies and may lead to good outcomes; however, consistent evidence in regard to RTP rates is lacking in the literature. ${ }^{8}$ Interventions for nonoperative treatment can include platelet-rich plasma (PRP) injection therapy, rest, and/or strengthening programs. ${ }^{9-12}$ As a partial UCL tear is a

Cascia and Uhl are with the Department of Rehabilitation Science, University of Kentucky, Lexington, KY, USA. Hettrich is with the Department of Orthopedic Surgery and Sports Medicine, University of Kentucky, Lexington, KY, USA. Cascia (Nicole.Cascia@uky.edu) is corresponding author. potential career-ending injury, it is imperative that the best treatment option is provided to these professional throwing athletes. Immediate and long-term RTP rates after conservative management of partial UCL injuries can help guide health care providers in deciding the best treatment in professional throwing athletes.

\section{Focused Clinical Question}

Is there evidence for successful RTP rates in professional baseball players following conservative treatment of a partial UCL injury?

\section{Summary of Search, "Best Evidence" Appraised, and Key Findings}

- The literature was queried for studies of level 4 evidence or higher that investigated RTP rates after nonoperative treatment of a UCL injury in professional baseball players.

- The literature search returned 11 articles that included professional baseball players as subjects. Five articles met the inclusion criteria by reporting RTP rates after a period of conservative treatment.

- Two articles were excluded because they were abstract only, leaving 3 articles for review. ${ }^{10-12}$ 


\section{Clinical Bottom Line}

Current evidence supports high success RTP rates (66\%-100\%) after conservative treatment of UCL injuries in professional baseball players.

\section{Strength of Recommendation}

Based on the low level of evidence, a grade $\mathrm{C}^{13}$ recommendation is given for high RTP rates following conservative management of UCL injuries in professional baseball players. Further investigation is warranted due to the limited quality of studies available due to both limited quantity and quality of evidence.

\section{Search Strategy}

\section{Terms Used to Guide Search Strategy}

- Patient/Client group: Professional baseball OR Elite baseball OR High-level baseball OR Major league OR Minor league

- Intervention: Nonoperative OR Rehabilitation OR Physical therapy OR Conservative OR Injection therapy

- Comparison: None

- Outcome: Return to sport or same level of play after partial $\overline{\mathrm{U} C L}$ injury (tear OR insufficiency OR sprain)

\section{Sources of Evidence Searched}

- CINAHL

- Academic Search Complete

- MEDLINE

- SPORTDiscus

- Health Source

- SAGE

- PubMed

\section{Inclusion and Exclusion Criteria}

\section{Inclusion Criteria}

- Reported RTP rates after nonoperative treatment

- RTP rates were reported in professional baseball players

- Magnetic resonance imaging confirmed diagnosis of a UCL injury

- Level 4 evidence or higher

- Reported in English language

- Literature search included dates between inception and 2018

\section{Exclusion Criteria}

- Did not report separate RTP rates on professional baseball players

- No evidence for conservative management

- Abstract only

\section{Results of Search}

Three relevant studies ${ }^{10-12}$ were identified and categorized in Table 1 (based on Levels of Evidence, Centre for Evidence-Based Medicine). ${ }^{14}$
Table 1 Summary of Study Designs of Articles Retrieved

\begin{tabular}{lcc}
\hline Level of evidence & Study design & References \\
\hline 4 & Retrospective & Dines et al ${ }^{10}$ \\
4 & Retrospective & Ford et al $^{11}$ \\
4 & Retrospective & Frangiamore et al $^{12}$ \\
\hline
\end{tabular}

\section{Best Evidence}

Studies selected for inclusion in this critically appraised topic are listed in Table 2. The 3 studies included were identified as the best evidence and selected as the most appropriate given the inclusion and exclusion criteria and focused clinical question. The Downs and Black ${ }^{15}$ checklist was used to assess the quality index of the studies. This checklist has been validated and is used to assess methodological quality of nonrandomized studies. ${ }^{15}$

\section{Implications for Practice, Education, and Future Research}

Previous research has explored nonoperative treatment outcome rates of partial UCL injuries in throwing athletes, ${ }^{16}$ but only the articles ${ }^{10-12}$ reviewed in this critically appraised topic reported RTP rates in professional baseball players. The results from this appraisal support nonoperative treatment as a successful treatment option for professional baseball players with a partial UCL tear with a grade 1 having the most success. ${ }^{10-12}$ The most important clinical implication proposed by all 3 articles is that UCL grade, location, and severity can be potential predictors of successful RTP in professional baseball players. It is also highly suggestive that magnetic resonance imaging alone cannot provide substantial enough information for treatment making decisions in professional baseball players. ${ }^{11,17}$ Rehabilitation strategies after injury should also be considered in the success of RTP but consistency lacks in transparency and reporting of interventions. ${ }^{10-12}$ In the articles reviewed, history, physical examination, and magnetic resonance imaging results were all considered in the methods for UCL injury diagnosis. ${ }^{10-12}$ Ford et al ${ }^{11}$ reported the highest RTP of $100 \%$ for grade 1 tears. High-grade tears had reported rates of $94 \%{ }^{11}$ (grade 2B) and as low as 66\%. ${ }^{12}$ Dines et al ${ }^{10}$ and Ford et $\mathrm{al}^{11}$ reported that proximally located tears resulted in higher RTP rates when compared with distally located tears but did not specify tear location for the professional baseball players in the study as an outcome variable. Frangiamore et $\mathrm{al}^{12}$ emphasized that tear location should be strongly considered and discussed with throwers when deciding between operative and nonoperative management of a UCL injury, with proximal tears resulting in higher odds of success. Furthermore, Frangiamore et al $^{12}$ found that high-grade tears were more likely to fail nonoperative management.

Platelet-rich plasma injections were used as a conservative treatment in one of the studies. ${ }^{10}$ The author concluded a successful RTP rate (66\%) with the use of PRP. ${ }^{10}$ The professional baseball players in the study had failed previous conservative treatment, and the PRP injections were given in conjunction with a rehabilitative program. After injection, the athlete refrained from throwing and followed a progressive stretching and strengthening program for 4 to 6 weeks before starting an interval throwing program. ${ }^{10}$ This provides evidence for both rehabilitation and injection therapy as a nonoperative treatment strategy in partial thickness UCL tears. Ford et $\mathrm{al}^{11}$ utilized modalities (electrical stimulation, soft tissue massage, 


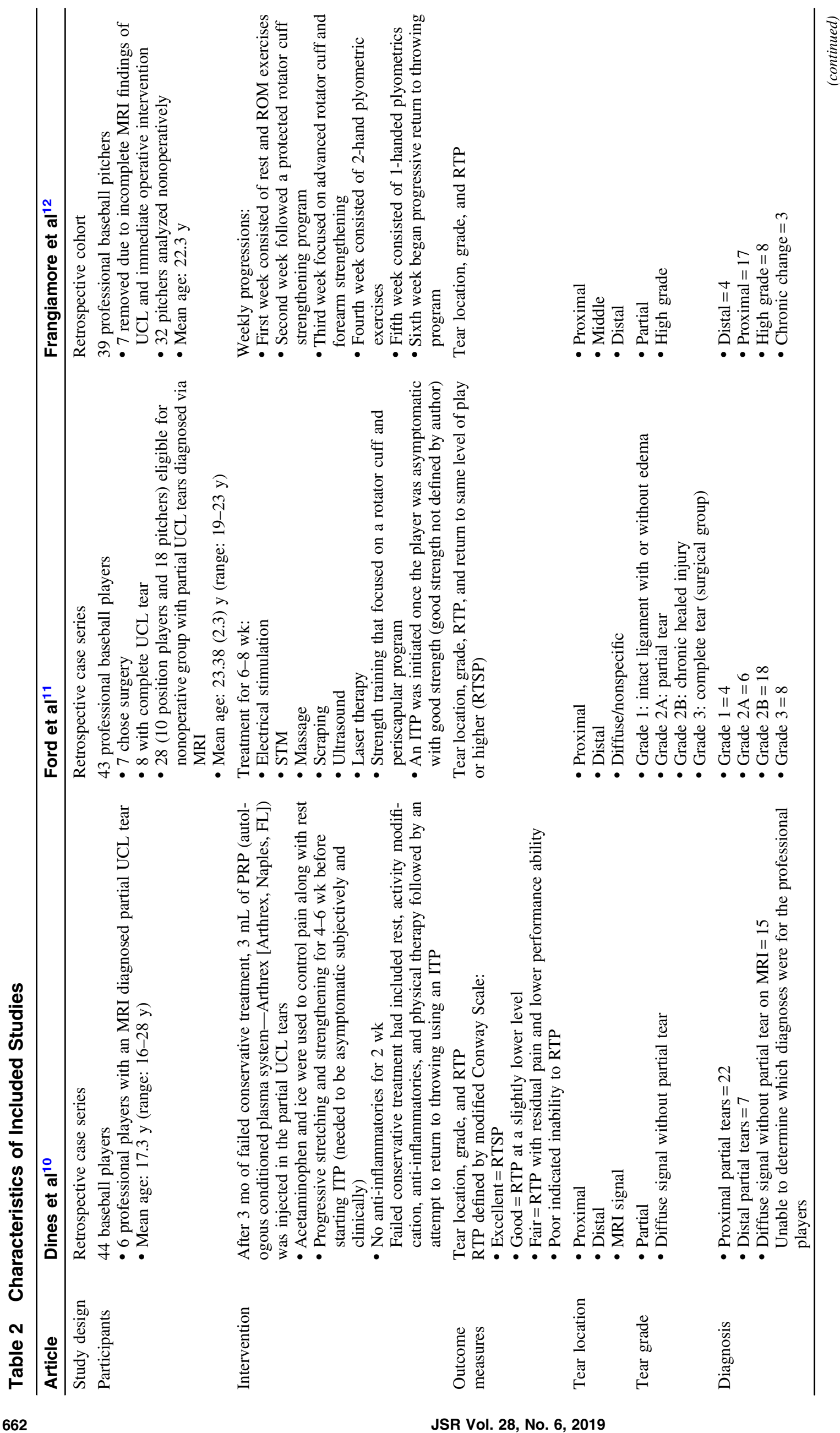




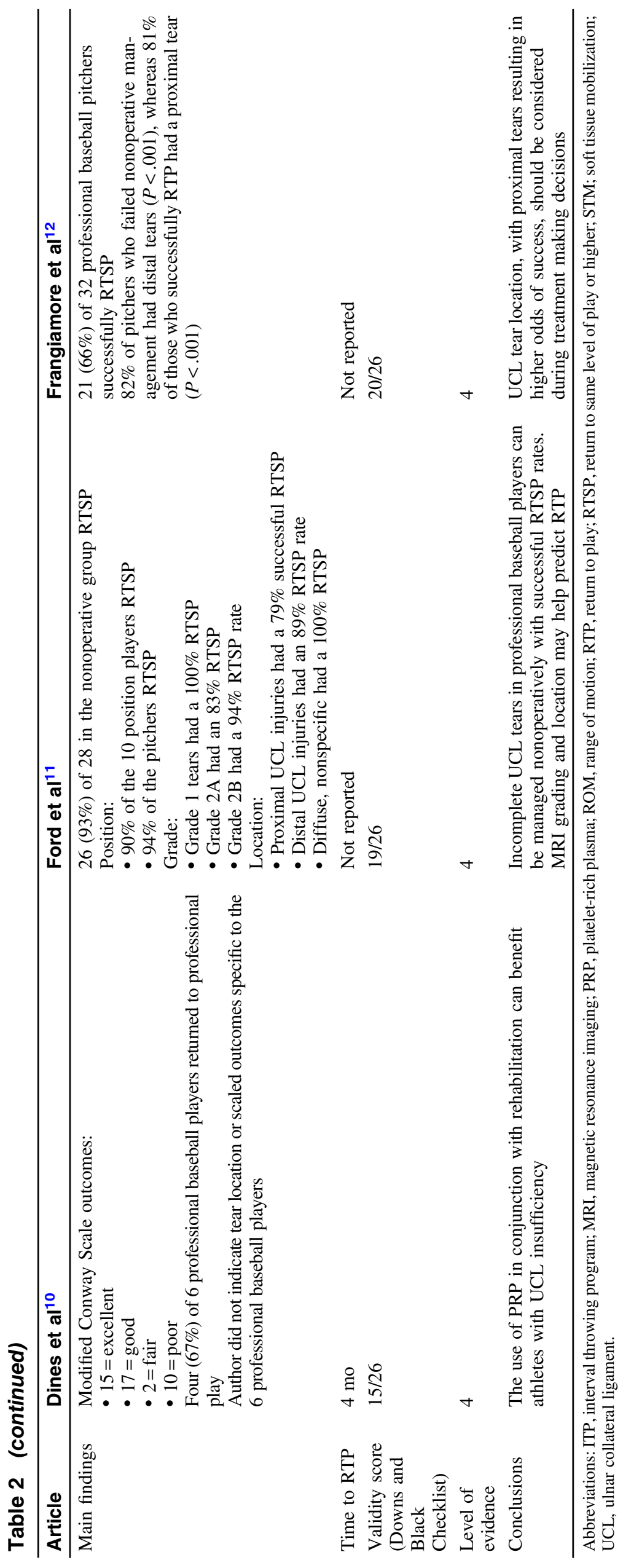


scraping, ultrasound, and laser therapy) for 6 to 8 weeks followed by a strength training program that consisted of a focused rotator cuff and periscapular program. An interval throwing program was implemented once the player was asymptomatic, had good strength, and was not based on a specific time frame. Frangiamore et $\mathrm{al}^{12}$ was the only author who reported weekly descriptions of their nonoperative treatment intervention. Week 1 consisted of rest and range of motion exercises, week 2 followed a rotator cuff strengthening program, and week 3 consisted of advanced rotator cuff and forearm strengthening. Weeks 4 and 5 consisted of 2- and 1-hand plyometric exercises, respectively. Patients then began a progressive return to throwing program at the beginning of week 6 . As part of the last phase in their nonoperative protocol, all 3 authors had consistently reported an interval throwing program. Overall, if a complete UCL tear was diagnosed, surgery was indicated. Greater variations existed in treatment methods when a partial UCL tear was diagnosed. ${ }^{10-12}$

We acknowledge limitations of this critically appraised topic. First, all studies ${ }^{10-12}$ were retrospective in design, which limits the quality of data and results for interpretation. A second limitation is noted in the inability to generalize each of the nonoperative interventions. Each conservative treatment strategy was different, making it difficult to pool results or make recommendations on the best strategy. One of the authors ${ }^{10}$ used an RTP evaluation tool (Conway Scale) during follow-up examinations to measure outcomes, but this scale has not yet been validated. This lack of scale validation may weaken the outcome measures classified following their intervention. In addition, Dines et al ${ }^{10}$ did not define "conservative treatment failure" in the professional baseball players who later received PRP injections.

There are currently no prospective studies in the literature for professional baseball players that evaluate RTP rate or success of RTP after nonoperative treatment in partial UCL tears. The paucity of literature available on this topic makes it challenging to properly determine if high-level athletes can be successfully treated nonoperatively. ${ }^{11}$ Given the rise in UCL surgical rates, future areas of research should focus on following professional baseball players with UCL injuries prospectively and over a longer period of time to see if the treatments provide long-term ability to RTP. Studies that incorporate measures of RTP rates after nonoperative management are paramount due to the foundational support they provide in treatment decisions and can further justify or support a conservative treatment plan. In addition, future UCL research should work toward formulating a treatment algorithm for decisions on operative and nonoperative management.

\section{Acknowledgment}

There are no conflicts of interest for any authors.

\section{References}

1. Erickson B, Gupta A, Harris J, et al. Rate of return to pitching and performance after Tommy John surgery in Major League Baseball pitchers. Am J Sports Med. 2014;42(3):536-543. PubMed ID: 24352622 doi:10.1177/0363546513510890

2. Makhni E, Lee R, Morrow Z, Gualtieri A, Gorroochurn P, Ahmad C. Performance, return to competition, and reinjury after Tommy John Surgery in Major League Baseball pitchers: a review of 147 cases. Am J Sports Med. 2014;42(6):1323-1332. PubMed ID: 24705898 doi:10. $1177 / 0363546514528864$
3. Erickson B, Chalmers P, Bush-Joseph C, Verma N, Romeo A. Ulnar collateral ligament reconstruction of the elbow: a systematic review of the literature. Orthop J Sports Med. 2015;3(12):2325967115618914. PubMed ID: 26740956 doi:10.1177/2325967115618914

4. Cain E Jr, McGonigle O. Return to play following ulnar collateral ligament reconstruction. Clin Sports Med. 2016;35(4):577-595. PubMed ID: 27543400 doi:10.1016/j.csm.2016.05.004

5. Wilson A, Pidgeon T, Morrell N, DaSilva M. Trends in revision elbow ulnar collateral ligament reconstruction in professional baseball pitchers. J Hand Surg Am. 2015;40(11):2249-2254. PubMed ID: 26328904 doi:10.1016/j.jhsa.2015.07.024

6. Mahure S, Mollon B, Shamah S, Kwon Y, Rokito A. Disproportionate trends in ulnar collateral ligament reconstruction: projections through 2025 and a literature review. J Shoulder Elbow Surg. 2016;25(6):10051012. PubMed ID: 27197888 doi:10.1016/j.jse.2016.02.036

7. Hurwit D, Garcia G, Liu J, Altchek D, Romeo A, Dines J. Management of ulnar collateral ligament injury in throwing athletes: a survey of the American Shoulder and Elbow Surgeons. J Shoulder Elbow Surg. 2017; 26(11):2023-2028. PubMed ID: 28941974 doi:10.1016/j.jse.2017.08.005

8. Watson J, McQueen P, Hutchinson M. A systematic review of ulnar collateral ligament reconstruction techniques. Am J Sports Med. 2014;42(10):2510-2516. PubMed ID: 24220014 doi:10.1177/ 0363546513509051

9. Podesta L, Crow S, Volkmer D, Bert T, Yocum L. Treatment of partial ulnar collateral ligament tears in the elbow with platelet-rich plasma. Am J Sports Med. 2013;41(7):1689-1694. PubMed ID: 23666850 doi:10.1177/0363546513487979

10. Dines J, Williams P, ElAttrache N, et al. Platelet-rich plasma can be used to successfully treat elbow ulnar collateral ligament insufficiency in high-level throwers. Am J Orthop. 2016;45(5):296-300. PubMed ID: 27552453

11. Ford G, Genuario J, Kinkartz J, Githens T, Noonan T. Return-to-play outcomes in professional baseball players after medial ulnar collateral ligament injuries: comparison of operative versus nonoperative treatment based on magnetic resonance imaging findings. Am J Sports Med. 2016; 44(3):723-728. PubMed ID: 26764237 doi:10.1177/0363546515621756

12. Frangiamore S, Lynch T, Vaughn M, Soloff L, Forney M, Styron J, Schickendantz M. Magnetic resonance imaging predictors of failure in the nonoperative management of ulnar collateral ligament injuries in professional baseball pitchers. Am J Sports Med. 2017;45(8):17831789. PubMed ID: 28398820 doi:10.1177/0363546517699832

13. Ebell M, Siwek J, Weiss B, et al. Strength of recommendation taxonomy (SORT): a patient-centered approach to grading evidence in the medical literature. J Am Board Fam Pract. 2004;17(1):59-67. PubMed ID: 15014055 doi:10.3122/jabfm.17.1.59

14. Oxford Centre for Evidence-Based Medicine. http://www.cebm.net/ index.aspx?o=5653

15. Downs S, Black N. The feasibility of creating a checklist for the assessment of the methodological quality both of randomised and nonrandomised studies of health care interventions. J Epidemiol Community Health. 1998;52:377-384. PubMed ID: 9764259 doi:10.1136/ jech.52.6.377

16. Rettig A, Sherrill C, Snead D, Mendler C, Mieling P. Nonoperative treatment of ulnar collateral ligament injuries in throwing athletes. Am J Sports Med. 2001;29(1):15-17. PubMed ID: 11206249 doi:10. 1177/03635465010290010601

17. Kooima C, Anderson K, Craig J, Teeter D, Van Holsbeeck M. Evidence of subclinical medial collateral ligament injury and posteromedial impingement in professional baseball players. Am J Sports Med. 2004;32(7):1602-1606. PubMed ID: 15494322 doi:10.1177/ 0363546503262646 